\title{
A Field Study of Haul Truck Operations in Open Pit Mines
}

\author{
Patrick Stahl, Birsen Donmez, Greg Jamieson \\ Mechanical and Industrial Engineering, University of Toronto
}

\begin{abstract}
This paper presents findings from a field study of the operation of haul trucks in two open pit gold mines. Qualitative results relevant to the haul truck operator's work environment are presented, and the human factors challenges of the work are identified. Three specific issues that stood out from the study are discussed in detail. First, fatigue is identified as a major contributing factor to crashes and overall performance in open pit traffic, heightened by the specific work characteristics of a haul truck operator. Second, negative transfer is discussed as it interferes with the adaptation from one truck type to another: a consequence of inconsistent controls across different truck brands. Third, the under utilization of and the general posture of suspicion towards the dispatch system are reported, with a list of potential reasons related to automation characteristics.
\end{abstract}

\section{INTRODUCTION}

Efficient operation of haul trucks is crucial to mine productivity. The higher the average speed of a truck (within the limits determined by the traffic rules), the more material it will move. Simultaneously, maintaining constant speeds for extended periods, without substantial decelerations, will result in reduced maintenance costs and improved fuel efficiency.

Haul truck traffic also plays an important role in mine safety. In an analysis of the injuries in small open pit coal mines, haulage was identified as playing a significant role in both lost-time injuries and fatalities (Randolph, 1998). In general, Krowczyk (2004) attribute $24.5 \%$ of all fatal mine accidents to surface haulage, a number that rises to $37.9 \%$ when the focus is on surface mining only. Further, research has identified vertical jarring (sudden vertical impact) of the trucks while in forward motion as the primary cause of injury for haul truck operators (Santos, Porter, \& Mayton, 2010). While the causes for haul truck crashes can rarely be attributed to a single factor, they often involve a significant human performance component (Patterson \& Shappell, 2010; Randolph, 1998). Fatigue in particular has been identified as a major factor contributing to crashes in haul traffic (Orchansky, Worrall, Malean, \& Nebot, 2006; Santos, et al., 2010; Schutte \& Maldonado, 2003).

The majority of safety-related research in mining, including the research cited above, has concentrated on accident analysis. The results are often recommendations, such as pre-operation inspections (e.g., ensuring use of seatbelts, and clean and non-slippery surfaces to move on) and means to heighten the safety of critical haul phases (e.g., use of cameras or proximity sensors for stationary dumping and backing up) (Turin, Wiehagen, Jaspal, \& Mayton, 2001).

In contrast, this paper looks upon open pit traffic and haul trucks as an opportunity for human factors research and improved human factors practice in mines. This work should therefore be regarded as an initial assessment of the field.

\section{PRACTICE INNOVATION}

An exploratory field study was conducted in two open pit gold mines in July 2010. Six consecutive days were spent at each of the two mine sites (i.e., 12 days total):
- The first mine was relatively large, characterized by one extensive, central pit, with a high volume of traffic and numerous intersections.

- The second mine was much smaller, and laid out in a satellite pattern (that is, it consisted of several individual pits that were geographically separated). Overall, the traffic in the second mine was significantly lower than in the larger mine, and consisted of hauls on long roads with few intersections.

The differences in mine characteristics pose disparate challenges to haul truck operators. In the smaller mine, the intersections were very sparse and the hauls could be extremely long as a consequence of the physical setup of the mine. Especially at night, there was almost no other traffic on the haul roads. Consequently, this smaller mine provided us with an excellent opportunity to interview operators with respect to the effects of extended periods of low stimulation and high fatigue.

The larger mine in contrast was characterized by significantly higher traffic on a tighter network of haul roads with numerous intersections. Drivers here regularly met other traffic and had to stop at intersections. Consequently, drivers could be observed under a higher taskload. A partially automated dispatch system was used in this mine to generate truck assignments (while the smaller mine was in the process of implementing one). Thus, the larger mine enabled us to observe operator attitudes towards this dispatch system (a commercial third party system). Different brands of trucks were used across and within these two mines, which enabled us to evaluate a range of truck cabins.

Due to the inability to build on prior research, the approach in this field study was very much exploratory and ethnographic in nature. The goal was to analyze and understand the work process of a haul truck operator, and generate suggestions for future research topics. The research was undertaken by observations as well as informal and semistructured interviews. Interviews were conducted with dispatch engineers, equipment maintenance engineers, foremen, and shovel operators. The researchers accompanied haul truck operators during their shifts, thereby developing a first-hand understanding of the work process and its 
challenges. During these observations, there was also ample opportunity for semi-structured and informal interviews. A total of 19 haul truck operators were directly observed by three researchers. Twelve observations took place in the smaller mine, three of which were undertaken during nightshifts. In the larger mine, seven haul truck operators, three dispatch engineers, and one shovel operator were observed during the day. Sessions with each operator ranged between 45 to 150 minutes.

Notes were taken during observations, and interviews were voice-recorded whenever possible. To capture the dynamics of the situations and analyze them later, video was also used, both in the cockpits (to record the drivers' interaction with the control elements) as well as in the pit (to better understand the interaction among vehicles at intersections and at loading and dumping sites).

\section{FINDINGS \& DISCUSSION}

\section{The Haul Cycle}

Haulage in open pit mines is highly cyclical in nature (Figure 1). A haul truck is initially assigned to a specific shovel or loader. The operator drives to that assigned position and loading of the truck begins. Depending on the load type, the truck then gets assigned to a specific site to unload. If it is loaded with waste, it goes to a dump; if it is loaded with ore, it goes to a processing site specific to the particular ore type. This phase of the cycle is called the haul. The operators spend the majority of their time in the haul phase. The unloading begins when the truck reaches the assigned destination. From here, the haul cycle starts again.

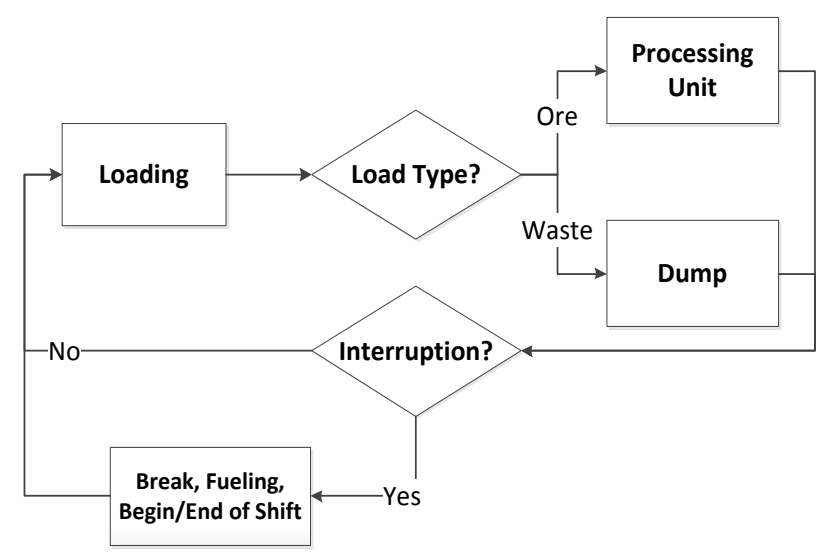

Figure 1: The Haul Cycle

While in the cabin, the operator's primary task is the navigation of the truck, which can be described according to a general model of information processing (Wickens \& Hollands, 2000). The drivers perceive the current state of the truck and the environment, mainly through visual cues, but also via haptic (vibrations from the truck itself and the road it is travelling on) and auditory (engine and brake sounds) channels. The operator's assessment of the truck's state within the environment, coupled with operator intentions, guides response selection and control input. This process can be subsumed as vehicle control.

In addition to vehicle control, the driver also has to perform other tasks, which mainly involve communicating with other parties in the mine to exchange information and/or plan specific actions. These tasks, which can be categorized as communication and information management, involve the use of the radio and the touch-screen dispatch interface in the cabin. Operators in the larger mine, for example, received their assignments through their dispatch displays, and therefore had to frequently monitor this display for changes in assignments, and interact with it to update their truck's state (e.g., to confirm a completed loading process). Another example observed was the coordination of multiple vehicles working in close proximity, carried out via communication on the radio. It is noteworthy that both vehicle control and communication and information management often have to be carried out in parallel, thereby potentially creating conflicting demands.

The demand imposed upon the operator by the primary task of vehicle control varies significantly over the phases of the haul cycle. During the loading process, operators are merely sitting in an idling truck, and there is no need to actively engage in vehicle control. However, the initial manoeuvring to correctly position the truck next to the shovel is a particularly challenging task for a haul truck operator. Drivers have to back up next to a moving shovel, and the large size of both machines results in the need for skilful estimation of distances with little visual feedback. The operators depend solely on two rear-view mirrors to perceive the position of the truck relative to the shovel. During this task, operators often ceased to carry out any secondary tasks, including interactions with the researcher. The cessation of all secondary tasks suggests not only high workload, but likely the reaching of maximum working memory capacity.

The initial manoeuvring for the loading task contrasts significantly with the haul phase, in which the operators' workload is relatively low. In particular, in the smaller mine, operators often kept the truck at a constant speed for long periods of time, with only minimal steering. Operators in this phase had an easy time carrying out communication and information management tasks, conversing with the researcher, and even demonstrating the various features of the trucks.

The unloading phase was observed to require high concentration again, although it was noticeably less complicated than manoeuvring next to the shovel due to the driver having more physical room, less need for precision in manoeuvring, and the aid of a camera filming the rear of the truck (the latter being an aid not universally available but reserved to specific, rather modern models with large payloads).

Two natural work design implications follow from the above assessment. First, during the phases of high workload, namely the manoeuvring required for both loading and unloading, no further tasks should be imposed upon the operators. Second, an opportunity for improvement is revealed: technical aids and automation can be helpful to the operator to carry out these high workload tasks, which require 
a high level of skill and precision. Potential research should therefore investigate the feasibility of technical aids for the task of pulling in next to a shovel.

For the actual haul phase of the haul cycle, the opposite is true. Tasks that are not crucial to vehicle control can be carried out during this phase of relatively low demand. Technical aids to further reduce workload here may even be counterproductive. Previous research suggests an inverted-U shape between arousal/workload level and performance (Hebb, 1955; Proctor \& Van Zandt, 2008; Yerkes \& Dodson, 1908), indicating a decrease in performance with both low and high levels of arousal. With regard to attention, it has been established that vigilance decrement occurs when low arousal is experienced for extended periods of time (Parasuraman \& Davies, 1976). Thus, a reduction in demand due to technical aids may lead to reduced arousal, which may in turn lead to vigilance decrements. Low arousal may also amplify fatigue (Williamson, 2009), which is discussed in the following section.

\section{Fatigue}

Fatigue has been defined as "a state of muscles and the central nervous system in which prolonged physical activity or mental processing, the absence of sufficient rest, leads to insufficient capacity or energy to maintain the original level of activity and/or processing." (Soames Job \& Dalziel, 2001). Particularly for driving it has been characterized as a progressive withdrawal of attention from road and traffic demands (Brown, 1994). Williamson uses this characterization as well, and argues based on arousal theory that one possible cause for fatigue may lie in "high monotony of stimulus presentation" (Williamson, 2009, p. 385). Within this theoretical perspective, the following section explains why the work environment of a haul truck operator in particular is prone to monotonous stimulus presentation, and consequently fatigue.

Mining in general is characterized by work in long shifts. For the two mines observed, the haul truck operators worked four consecutive days in a week, with a 12-hour shift each day. During a 12-hour shift, two short breaks of 15 minutes each were common among the drivers. In addition, operators often spent significant time in their daily commute, due to the typically remote mine locations. The drivers interviewed commuted between two to five hours every day.

The resulting picture is one of operators who have to carry out a highly monotonous work routine reliably for 12 hours, and who spend most of their shift in the low-arousal haul phase. In particular, the circumstances in night shifts may further amplify the high risk associated with fatigue-related crashes. These circumstances include lack of light (which reduces visual stimuli), circadian rhythm, and the loneliness of the "graveyard shift" (Lal \& Craig, 2001). Apart from the haul trucks, the roads were deserted at night.

Operators often described falling into a state of drowsiness during night shifts and feeling "as if everything happened automatically". While some of the drivers preferred the night shifts due to being less busy, all agreed on the high danger of "losing grip on what you're doing", and of falling asleep, especially in the early morning hours right before sunrise. When asked what they did in order to prevent fatigue, operators responded by explaining various strategies they use. Listening to music via radio and CDs, conversing with other drivers on the radio, eating, and drinking were the recurring examples. As Williamson (2009) points out, such strategies indicate that in specific situations, additional distractive stimuli may actually have a positive, rather than a negative influence. Consequently, a reduction of workload in such situations might have the counterproductive effect of reducing stimuli and amplifying the effects of fatigue.

While the difficulty in maintaining vigilance is a consequence of job characteristics and cannot easily be changed, the fatigue resulting from long shifts may be easier to address. In particular for night driving, a reduction of shift durations and an increase in the frequency of breaks should be considered.

\section{Changing Equipment and Negative Transfer}

When asked about their preferences across the different types of trucks, multiple operators commented on the difficulties of getting accustomed to new equipment. The smaller mine in particular used a mixed fleet of trucks from two leading manufacturers. The differences in handling characteristics and controls between the brands were substantial enough that operators specifically requested to be assigned to only one brand. While in general every driver had one standard truck to which she was assigned, frequent changes in dispatch schedules (e.g., due to illness of operators) would require drivers to switch trucks.

Several operators commented on having problems due to the significant differences in the setup and controls of trucks. The reason for these problems in many cases is inconsistent design of control elements across the types of trucks, and the consequent negative transfer (Wickens \& Hollands, 2000).

One of the biggest differences between the two brands observed in the study is the braking system and its controls. One brand of trucks uses electrical motors to accelerate, and is equipped with energy-efficient, electrical-dynamic retarders. These dynamic retarders can be operated with either a lever next to the steering wheel, or the usual brake pedal, but are only effective above a specific speed. Below that speed, a conventional, hydraulic-actuated service brake has to be used. Thus, the dynamic retarders cannot alone bring the truck to a complete stop. The service brake is controlled solely by the pedal, and activated only if the pedal is pressed nearly all the way, and passes a momentary point of resistance. Up to this point of resistance, only the dynamic retarders respond. The pedal, therefore, functions in a very similar fashion to that of a regular car. That is, braking power can be continuously increased by applying more force to the pedal.

The fully hydraulic brakes of the second brand of trucks, however, are controlled in a very different manner. The only way of continuously regulating braking power here is the retarder lever on the steering wheel. In contrast, the brake pedal functions in binary fashion; that is, it can only apply full braking power or no power at all. Consequently, a driver switching from a truck with only hydraulic brakes to one with 
dynamic retarders can easily forget that the truck will not come to a complete stop by use of just the lever. Likewise, the operator switching in the opposite direction runs the danger of using the brake pedal to decelerate the truck, not anticipating the sudden application of full brake force.

While the larger mine did not use the models with the binary brake pedal, another type of truck observed here (also electrical) had yet another set of braking controls. This type of truck uses two separate pedals to control the dynamic retarders and the hydraulic service brake. Moreover, in comparison to the former brand of electrical trucks, the orientation of the gearbox was reversed.

Eliminating such negative transfer problems would involve establishing a consistent, ergonomic design of control elements across all different truck models. Such a dramatic intervention is not realistic, therefore mine managers should be aware of these issues, strive to avoid situations in which haul truck operators have to switch between different truck models, and take this factor into account when extending their haul fleet.

\section{Automation in Mine Traffic: Dispatch}

With few exceptions - one mine in Chile, for example, uses fully automated trucks (Rio Tinto, 2009) - automation in haul traffic seems to be limited. In the two mines we observed, the only complex automated system was the dispatch system, which assigns trucks to specific loading and unloading locations. Once set up with respect to the specific state of the mine (the exact locations of the individual pits change slowly with the progress of the mining operation), the system works in a highly automated fashion. The only human operator with privileges to override the assignments is the dispatch engineer. The truck operators have no direct means to influence their assignments.

The general attitude of operators towards the dispatch system was neutral at best, and frequently quite negative. Operators described the system as inflexible, as a "hassle to operate", and often reported not concurring with the system. One particular operator, for example, ignored the assignment to a specific dump as it was displayed on the dispatch display, and approached a closer dump location. While driving there, she used her cell phone to call the dispatch engineer and requested reassignment to the geographically closer dump. The mismatch between the operator and the automation may be due to two reasons. If the dispatch algorithm was not accurate and the operator had assessed the situation more properly, then this case can be viewed as an example of appropriate disuse (Lee, 2006; Lee \& See, 2004; Parasuraman \& Riley, 1997). Alternatively, the dispatch algorithm may have been factoring in information that the operator was not aware of (such as the desired blend of materials at specific dumps) and creating a global optimum. Regardless of the reason, the observed scenario reveals a discrepancy in the information exchange between the two parties.

The dispatch interface was also used to implement a system to monitor truck speed to ensure adherence to the maximum allowable speed, as well as a GPS-based proximity warning system. Both systems were perceived negatively. The speed advisory system was simply perceived as limiting with respect to the truck's capabilities on long, straight sections of the haul, where drivers felt they could maintain higher speeds. The proximity warning system had first been implemented to provide an auditory alert to the operators when there was a vehicle in close proximity. The operators interviewed reported receiving alerts when there was no apparent danger. Thus, the operators perceived the proximity warning system as a nuisance and ignored it altogether. The remedy applied was to replace the auditory warning with a visual warning presented on the dispatch display. Thus, the system was activated just as often as before, however, it became easier for the operators to ignore.

Yet, the primary function of the dispatch system is the assignment of equipment. If we describe this process with respect to the stages of automation proposed by Parasuraman, Sheridan, and Wickens (2000), it is evident that the information acquisition stage is occurring with almost no input from haul truck operators; they need only confirm being loaded or being empty again. The stages of information analysis and decision selection are also completely automated, with no driver input. The decision about the next assignment is simply presented to the haul truck operator on the dispatch display. In contrast, the action implementation is left to the operator.

By not taking into account sudden changes in road condition, the assessment of the dispatch algorithm may not always be the best one, and truck operators cannot influence or override the system. Operators' frustration with the dispatch system in general may stem from the dynamics of this particular way of implementing automation. Numerous discussions with the drivers about the faults of the current system and possible improvements indicated that few operators were fundamentally opposed to automation, but that they did not want to feel "out of the loop". They wanted a system that worked well and that they could influence.

The question becomes whether the solution to the problems presented here lies in allowing the operators to actively input information into the dispatch algorithm. Future research should investigate the influence of different levels of automation on compliance with such an automation aide. Specifically, with respect to information acquisition, operators may prove to be a valuable source of information, since they can provide first-hand knowledge about road and weather conditions, or the occurrence of other sudden changes that require replanning of the assignments (e.g., road blockages). Due to unpredictable changes in the environment, but also simply due to the fact that the dispatch system (just like any other type of automation) cannot possibly be accurate in every situation, operator input to and the ability to override the system may enhance the overall system performance.

\section{CONCLUSION}

A field study on haul traffic in open pit mines was conducted. Three issues relevant to human factors practice and research were identified: 
- Fatigue: The combination of long shifts and a job characterized by high repetitiveness and monotony results in fatigue being a major problem in this environment. Apart from an organizational approach to the problem, (i.e., an adjustment of work schedules and shift lengths), especially an investigation of the effect of an artificial increase in stimuli seems promising. Secondary tasks, and specifically the interaction with the dispatch system, could be modulated to increase stimuli during low-arousal phases, and avoid distracting the operator during phases of high demand.

- Negative transfer: Operators frequently have to switch between different truck types. Inconsistent design of controls across vehicles facilitates negative transfer. While this problem can only be addressed through an avoidance of vehicle switches for existing truck types, ergonomic analyses of crucial control elements (such as brakes) can be undertaken to determine a selfexplanatory, clear layout.

- Lack of trust in and disuse of automation: The automation characteristics have resulted in a generally negative attitude towards the dispatch system. Specifically, the exclusion of the operator from the dispatch decision making process may foster this attitude, so that future studies could investigate this effect by altering the extent of operator involvement. Allowing operator information input on the first stage of automation may heighten situation awareness, and have a positive impact on trust.

These three issues were discussed in detail and implications for human factors practice and research were identified. While all three areas require further attention from the human factors community, there is ample opportunity to influence the design and implementation of appropriate automation in mine traffic. The automation adopted so far is limited compared to other domains frequently studied by human factors researchers, creating the opportunity for the human factors community to guide acquisition and implementation decisions before they are made.

A general limitation of this research was the qualitative and exploratory nature of the field study conducted. Given that this was an initial attempt to identify human factors research and practice opportunities in this domain, a detailed plan was not put in place to address a priori research questions. A follow up field study will specifically focus on characterizing the interactions between the automated dispatch system and various human operators (e.g., dispatch engineer, drivers). We will conduct focus groups, administer questionnaires, and thus acquire further qualitative as well as quantitative data, in order to build a better understanding on facilitating human automation collaboration.

\section{ACKNOWLEDGEMENTS}

The funding for this research effort was provided by the MITACS Accelerate Internship and the Barrick Gold Corporation. We gratefully acknowledge the support of the Barrick Gold Corporation and the Queen's University Mining Department in the conduct of this field study. Many thanks to
Dr. Joshua Marshall for his insightful comments on the automation section.

\section{REFERENCES}

Hebb, D. O. (1955). Drives and the C. N. S. (conceptual nervous system). Psychological Review, 62, 243-254.

Krowczyk, C. (2003). Haulage Fatalities at Surface Mines, 1999-2003. U.S. Deparment of Labor, Mine Safety and Health Administration, Program Evalution and Information Resources, Office of Injury and Employment Information.

Lal, S. K. L., \& Craig, A. (2001). A critical review of the psychophysiology of driver fatigue. Biological Psychology, 55, 173-194.

Lee, J. D. (2006). Human factors and ergonomics in automation design. In G. Salvendy (Ed.), Handbook of Human Factors and Ergonomics (3rd ed., 1570-1596). New Jersey: John Wiley \& Sons.

Lee, J. D., \& See, K. A. (2004). Trust in automation: designing for appropriate reliance. Human Factors, 46(1), 50-80.

Orchansky, D., Worrall, S., Malean, A., \& Nebot, E. (2006). Designing a user interface for improving the awareness of mining vehicle operators. Proceedings of the 13th International IEEE Annual Conference on Intelligent Transportation Systems, 1435-1441.

Parasuraman, R., \& Davies, D. R. (1976). Decision theory analysis of reponse latencies in vigilance. Journal of Experimental Psychology: Human Perception and Performance, 2, 569-582.

Parasuraman, R., \& Riley, V. (1997). Humans and automation: use, misuse, disuse, abuse. Human Factors, 39(2), 230-253.

Parasuraman, R., Sheridan, T., \& Wickens, C. D. (2000). A model for types and levels of human interaction with automation. IEEE Transactions on Systems, Man and Cybernetics - Part A: Systems and Humans, 30(3), 286-297.

Patterson, J. M., \& Shappell, S. A. (2010). Operator error and system deficiencies: analysis of 508 mining incidents and accidents from Queensland, Australia using HFACS. Accident Analysis and Prevention, 42, 1379-1385.

Proctor, R. W., \& Van Zandt, T. (2008). Human Factors in Simple and Complex Systems (2nd ed.). Boca Raton, FL: CRC Press.

Randolph, R. F. (1998). Injury analysis of Pennsylvania small surface coal mines. Proceedings of the 3rd Health and Safety Seminar for Small Mines, 83-92. University Park, PA.

Rio Tinto (2009). All under control. Rio Tinto Review Sept. 2009, 20-23.

Santos, B. R., Porter, W. L., \& Mayton, A. G. (2010). An analysis of injuries to haul truck operators in the US mining industry. Proceedings of the Human Factors and Ergonomics Society 54th Annual Meeting, 1870-1874. San Francisco, CA.

Schutte, P. C., \& Maldonado, C. C. (2003). Factors effecting haul truck driver alertness during the operation of haul trucks in South African mining industry. (SIM 020502). Safety in Mines Research Advisory Committee: CSIR mining technology.

Soames Job, R.F., Dalziel, J. (2001). Defining fatigue as a condition of the organism and distinguishing it from habituation, adaption, and boredom. In: P.A. Hancock \& P.A. Desmond (Eds.), Stress, Workload and Fatigue, 466-475. Mahwah: Lawrence Erlbaum Associates.

Turin, F. C., Wiehagen, W. J., Jaspal, J. S., \& Mayton, A. G. (2001). Haulage truck dump site safety: an examination of reported injuries. (2001124). US National Institute of Occupational Safety and Health.

Wickens, C. D., \& Hollands, J. G. (2000). Engineering Psychology and Human Performance (Third ed.). New Jersey: Prentice Hall.

Williamson, A. (2009). The relationship between driver fatigue and driver distraction. In A. R. Reagan, J. D. Lee \& K. L. Young (Eds.), Driver Distraction: Theory, Effects, and Mitigation, 383-392. Boca Raton: CRC Press.

Yerkes, R. M., \& Dodson, J. D. (1908). The relation of strength of stimulus to rapidity of habit-formation. Journal of Comparative Neurology and Psychology, 18, 459-482. 